OPEN ACCESS

Edited by:

Kian Mau Goh,

University of Technology Malaysia,

Malaysia

Reviewed by:

Xinjiong Fan,

Anhui Medical University, China

Hui Ni,

Jimei University, China

${ }^{*}$ Correspondence:

Jung-Hoon Yoon

jhyoon69@skku.edu

Specialty section:

This article was submitted to

Microbiotechnology, Ecotoxicology,

and Bioremediation,

a section of the journal

Frontiers in Microbiology

Received: 11 September 2019 Accepted: 19 December 2019

Published: 24 January 2020

Citation:

Park J-M, Kang C-H, Won S-M,

Oh K-H and Yoon J-H (2020)

Characterization of a Novel

Moderately Thermophilic

Solvent-Tolerant Esterase Isolated

From a Compost Metagenome

Library. Front. Microbiol. 10:3069.

doi: 10.3389/fmicb.2019.03069

\section{Characterization of a Novel Moderately Thermophilic Solvent-Tolerant Esterase Isolated From a Compost Metagenome Library}

\author{
Ji-Min Park', Chul-Hyung Kang ${ }^{1,2}$, Sung-Min Won ${ }^{1}$, Ki-Hoon Oh${ }^{3}$ and Jung-Hoon Yoon ${ }^{1 *}$ \\ 1 Department of Food Science and Biotechnology, Sungkyunkwan University, Suwon, South Korea, ${ }^{2}$ Green Chemistry \\ and Environmental Biotechnology Program, School of Science, University of Science and Technology, Daejeon, \\ South Korea, ${ }^{3}$ Korea Research Institute of Bioscience and Biotechnology, Daejeon, South Korea
}

A novel esterase, EstCS1, was isolated from a compost metagenomics library. The EstCS1 protein, which consists of 309 amino acid residues with an anticipated molecular mass of $34 \mathrm{kDa}$, showed high amino acid sequence identities to predicted esterases and alpha/beta hydrolases (59\%) from some cultured bacteria and to predicted lipases/esterases from uncultured bacteria. The phylogenetic analysis suggested that the EstCS1 belongs to the hormone-sensitive lipase family of lipolytic enzyme classification and contains a catalytic triad including Ser155-Asp255-His285. The Ser155 residue of the catalytic triad in the EstCS1 was located in the consensus active-site motif, GXSXG. Besides, a conserved HGGG motif placed in an oxyanion hole of the hormone-sensitive lipase family was discovered, too. The EstCS1 demonstrated the highest activity toward p-nitrophenyl propionate (C3) and caproate (C6) and was normally stable up to $60^{\circ} \mathrm{C}$ with optimal activity at $50^{\circ} \mathrm{C}$. In addition, an optimal activity was observed at $\mathrm{pH} 8$, and the EstCS1 possessed its stability within the $\mathrm{pH}$ range between 5 and 10. Interestingly, EstCS1 had an outstanding stability in up to $30 \%(\mathrm{v} / \mathrm{v})$ organic solvents and activity over $50 \%$ in the presence of $50 \%(\mathrm{v} / \mathrm{v})$ acetone, ethanol, dimethyl sulfoxide (DMSO), and N,N-dimethylformamide. The EstCS1 hydrolyzed sterically hindered tertiary alcohol esters of $t$-butyl acetate and linalyl acetate. Considering the properties, such as the moderate thermostability, stability against organic solvents, and activity toward esters of tertiary alcohols, the EstCS1 will be worthwhile to be used for organic synthesis and related industrial applications.

Keywords: metagenome, lipolytic enzyme, esterase, family IV, solvent tolerant

\section{INTRODUCTION}

Lipases (EC 3.1.1.3) and esterases (EC 3.1.1.1) are $\alpha / \beta$ hydrolases that are produced by a lot of microorganisms and eukaryotic organisms (Bornscheuer, 2002). Lipases catalyze the synthetic and hydrolytic reaction of slightly long-chain triacylglycerols and have quite low water solubility, whereas esterases that are partially soluble in water express a preference for the catalysis of shortchain triacylglycerols (Arpigny and Jaeger, 1999; Gupta et al., 2004). Lipolytic enzymes have the 
catalytic triad formed by Ser-Asp/Glu-His residues, and the Ser residue is generally conserved in the Gly-X-Ser-X-Gly pentapeptide motif (Bornscheuer, 2002). Lipolytic enzymes have drawn significant attention on account of useful and unique catalytic properties such as broad substrate specificity, organic solvent stability, position selectivity, and stereoselectivity, which is worthy of being used in agriculture, pharmaceutical, food, and fine chemical industries (Bornscheuer, 2002; Panda and Gowrishankar, 2005; Tutino et al., 2009).

Bacterial and metagenomic lipolytic enzymes have been classified into 17 families (families I-XVII) according to their biological attributes and amino acid sequence (Arpigny and Jaeger, 1999; Castilla et al., 2017). Among the 17 families showing bacterial lipolytic enzymes, family IV showed noticeable sequence similarity to mammalian hormone-sensitive lipases (HSLs), which play a momentous role in regulation of lipid metabolism (Yeaman et al., 1994; Arpigny and Jaeger, 1999; Kim, 2017). The genetic libraries were obtained directly from environmental sources without microbial culture in a laboratory by means of screening methods based on activity and sequence to effectively identify novel enzymes being used in various industries (Lee et al., 2006; Schmeisser et al., 2007; Kim et al., 2009; Uchiyama and Miyazaki, 2009; Berini et al., 2017). Especially, a number of lipases and esterases belonging to family IV have been recently isolated from various environmental sources since the development of metagenomic analyses (Oh et al., 2012; Choi et al., 2013; Privé et al., 2015; Kim, 2017; Huo et al., 2018; Jayanath et al., 2018; Noby et al., 2018).

Soil composting is a process of biological decomposition and humification of organic materials by microorganisms existing in the environment, and its temperature generally rises up to $80^{\circ} \mathrm{C}$ during the thermogenics period in the process. Thermophiles belonging to the genera Thermus, Bacillus, Hydrogenobacter, and Geobacillus have been commonly isolated from that compost (Beffa et al., 1996; Poli et al., 2006). Hydrolytic enzymes including cellulases, hemicellulases, proteases, phosphatases, arylsulfatases, and lipases are released by those microorganisms and play key roles in the composting process (Goyal et al., 2005). Therefore, the compost may be a favorable habitat to search for enzymes with thermostable characteristics.

While metagenomic genetic libraries were constructed from a compost of South Korea to screen novel thermostable lipolytic enzymes, some clones showing lipolytic activity were isolated. Among the genes subcloned, one lipolytic gene (designated estCS1) belonging to the family IV and indicating the strongest activity was chosen for further study. In this study, we describe methods and experimental results of cloning, expression, and biochemical characterization of this novel lipolytic enzyme.

\section{MATERIALS AND METHODS}

\section{Strains and Plasmids}

Escherichia coli strain EPI300-T1R (Epicenter) was used for the construction of a metagenomic library. E. coli DH5 $\alpha$ (Intron biotechnology) and BL21(DE3) (Invitrogen) were used as host strains for subcloning and overexpression of metagenomic gene retaining lipolytic activity, respectively. The pCC1FOS (Epicenter, United States) and pUC19 vectors were used for subcloning of the open reading frame encoding lipolytic activity. The pET22b $(+)$ vector (Novagen) was lastly used for cloning and overexpression.

\section{Construction of the Metagenomic Library and Screening of Lipolytic Genes}

Metagenomic DNA was extracted from a compost sample as previously described (Zhou et al., 1996). That genetic information was collected for the creation of metagenomic libraries with the CopyControl ${ }^{\mathrm{TM}}$ fosmid library production kit (Epicenter) in accordance with the manufacturer's instructions. For esterase/lipase screening based on enzymatic activities, transformants were incubated on Luria-Bertani (LB) agar media containing $1 \%(\mathrm{v} / \mathrm{v})$ emulsified tributyrin (C4) as a lipid substrate with chloramphenicol $(25 \mu \mathrm{g} / \mathrm{ml})$ and $0.5 \%(\mathrm{w} / \mathrm{v})$ gum arabic. Positive colonies forming clear halos on the agar media were singled out as candidates for novel lipolytic enzymes, and those genes were sequenced through a random shotgun sequencing method.

\section{Sequence Analysis}

The open reading frames encoding the esterase/lipase were compared with reference sequences that were retrieved from protein and nucleotide databases in the National Center for Biotechnology Information (NCBI). Sequence similarity was investigated through the Protein BLAST program at the NCBI, and multiple alignments were processed with sequences having high similarity by means of the Clustal W program. The phylogenetic tree was constructed using MEGA X program (Kumar et al., 2018) with 1,000 bootstrap replicates; numbers at branching points indicate the percentage of consensus.

\section{Cloning and Overexpression of the estCS1 Gene}

The putative esterase gene was amplified by PCR using forward and reverse primers ( $5^{\prime}$-GCGCATATGTCTATTCACCCA- $3^{\prime}$ and 5'-GGCCTCGAGCTTTTCACGA ATG-3'; the underlined letters indicate the $N d e I$ and $X h o I$ recognition sites, respectively). Ligation of the PCR fragment and the pET-22b(+) vector was carried out after they were cleaved with NdeI and XhoI. The recombinant plasmid DNA, designated pET-estCS1, was transformed to E. coli BL21 (DE3). The transformed E. coli was incubated in LB broth medium with ampicillin $(100 \mu \mathrm{g} / \mathrm{ml})$ at $37^{\circ} \mathrm{C}$. When the optical density of the culture attained an absorbance of $\sim 0.6$ at $600 \mathrm{~nm}, 0.5 \mathrm{mM}$ isopropylD-thiogalactopyranoside was added to induct the protein overexpression, and these $E$. coli cells were additionally incubated for $18 \mathrm{~h}$ at $21^{\circ} \mathrm{C}$. The cells were harvested by centrifugation and resuspended in binding buffer $(50 \mathrm{mM}$ Tris- $\mathrm{HCl}$ at $\mathrm{pH}$ 8.0 containing $300 \mathrm{mM} \mathrm{NaCl}$ ). Resuspended cells were then sonicated for disruption, and the crude cell lysate was centrifuged in $16,000 \mathrm{rpm}$ at $4^{\circ} \mathrm{C}$ for $30 \mathrm{~min}$. The supernatant including the cell extract was loaded on to a selective column packed with nickel-nitrilotriacetic acid (Ni-NTA) resin (QIAGEN GmbH). 
After washing the adsorbed resin using a buffer containing $2 \mathrm{mM}$ imidazole, the bound proteins having high affinity with a Ni-NTA were slowly eluted with a buffer featuring $250 \mathrm{mM}$ imidazole. Concentration of the eluted fractions was conducted by centrifugation in $5,000 \times g$ at $4^{\circ} \mathrm{C}$. The concentrated fractions were transferred to a gel filtration column (Superdex 200 10/300 GL; GE Healthcare) equilibrated with 50 mM Tris$\mathrm{HCl}$ buffer ( $\mathrm{pH} 8.0$ ) including $300 \mathrm{mM} \mathrm{NaCl}$ and consecutively separated at a flowrate of $0.5 \mathrm{ml} / \mathrm{min}$ using the BioLogic DuoFlow Chromatography System (Bio-Rad Laboratories). The target protein fraction showing the sharp peak in the gel filtration chromatography was analyzed through sodium dodecyl sulfate (SDS) polyacrylamide gel electrophoresis) in 12\% polyacrylamide gels.

\section{Biochemical Characterization of EstCS1}

The concentration of the enzyme was measured in accordance with the method of Bradford (Bio-Rad Protein Assay) with bovine serum albumin as a standard. The standard assay was performed by blending $10 \mathrm{mM}$-nitrophenyl butyrate (C4), ethanol, and $100 \mathrm{mM}$ GTA buffer (100 mM 3,3-dimethylglutaric acid, $100 \mathrm{mM}$ Tris-HCl, and $100 \mathrm{mM}$ 2-amino-2-methyl1,3-propanediol) as a mixture (1:4:95, by vol). During the reaction, the absorbance at $405 \mathrm{~nm}$ was continuously measured at regular intervals for quantifying the $p$-nitrophenol released from the $p$-nitrophenyl butyrate in the mixture using an Eon spectrophotometer (BioTek). One unit of enzyme activity was defined as the amount of enzyme required to produce $1 \mu \mathrm{mol}$ of $p$-nitrophenol per minute at $25^{\circ} \mathrm{C}$. The preference for $p$-nitrophenyl esters (pNPEs) as substrates (acetate, C2; propionate, C3; butyrate, C4; caproate, C6; caprylate, C8; caprate, C10; laurate, C12) was evaluated enzymatically through gauging the quantity of $p$-nitrophenol released during hydrolysis. To confirm the optimal reaction temperature, the reaction mixtures were incubated with no shaking for $10 \mathrm{~min}$ at varied temperatures $\left(10-80^{\circ} \mathrm{C}\right)$. Thermostability of EstCS1 was ensured by preincubation in the $100 \mathrm{mM}$ GTA buffer $(\mathrm{pH} 9.0)$ for $1 \mathrm{~h}$ under the same temperature conditions as the previous experiment, and then, the residual activity was gauged at room temperature. The $\mathrm{pH}$ related to optimal activity of the enzyme was ensured at $25^{\circ} \mathrm{C}$ in the $100 \mathrm{mM}$ GTA buffer using $\mathrm{pH}$ range of 4.0-11.0. Information of the $\mathrm{pH}$ stability was obtained by incubating the enzyme with $100 \mathrm{mM}$ GTA buffer ( $\mathrm{pH} 4.0-$ 11.0 ) for $16 \mathrm{~h}$ at $4^{\circ} \mathrm{C}$, and residual activity was determined at room temperature $\left(25^{\circ} \mathrm{C}\right)$. The analyses related to the optimal activity and the $\mathrm{pH}$ stability under various $\mathrm{pH}$ conditions were conducted simultaneously with the control test. Four mixtures containing $100 \mathrm{mM}$ GTA buffer ( $\mathrm{pH} 4.0-11.0$, at intervals of 1.0 $\mathrm{pH}$ unit) and EstCS1 were designated control, reaction 1, reaction 2 , and reaction 3, respectively. After reaction under the same conditions with $100 \mu \mathrm{l} 2 \mathrm{~N} \mathrm{NaOH}$, the absorbance at $405 \mathrm{~nm}$ was measured for quantifying $p$-nitrophenol released from the blended substrate ( $p$-nitrophenyl butyrate) in the mixture using an Eon spectrophotometer (BioTek). After that, the final value was derived by comparing the mean of the measurements of the one to three reactions with the control figure to take into account the automatic-hydrolyzation of the substrate.

\section{Inhibitory Effect of Metal lons, Detergents, Inhibitors, and Organic Solvents on the Activity of EstCS1}

To measure the effect of additives on the activity of EstCS1, various detergents and metal ions were used for incubation with purified and concentrated enzyme in the $100 \mathrm{mM}$ GTA buffer $(\mathrm{pH} 9.0)$ at $30^{\circ} \mathrm{C}$ for $1 \mathrm{~h}$. Following incubation, the remaining activity was gauged under standard assay conditions. The inhibitory effect of several materials $(1,5$, and $10 \mathrm{mM}$ concentrations) such as phenylmethylsulfonyl fluoride, dithiothreitol (DTT), ethylenediaminetetraacetic acid, and 2mercaptoethanol was investigated after incubation with EstCS1 at $30^{\circ} \mathrm{C}$ for $1 \mathrm{~h}$. The stability test of EstCS1 against organic solvents was performed with 10, 20, 30, and 50\% (w/v) of acetonitrile, methanol, dimethyl sulfoxide (DMSO), ethanol, acetone, 2-propanol, and N,N-dimethylformamide (DMF) in the same condition as it was used for the analysis of inhibitor.

\section{D Structural Prediction of EstCS1}

To predict a more reliable structure of EstCS1, two "web-based protein structure prediction systems," SWISS-MODEL ${ }^{1}$ and I-TASSER $^{2}$, were used. In the case of the SWISS-MODEL system, 50 reference enzymes with structures registered in the protein data bank $(\mathrm{PDB})^{3}$ by the identity score were sorted, and then, tertiary structures of EstCS1 were built. The information of the best model including sequences, distances, and $x y z$ coordinates of amino acids in EstCS1 was saved in PDB file format. In addition, multiple alignment among top 5 reference templates with EstCS1 through ESPript 3.0 $0^{4}$ was performed. Then, the AntheProt $3 \mathrm{D}$ viewer (version 1.4.2) to visualize the pdb file in the ribbon and the alpha trace form was used. The catalytic triad and the oxyanion hole of EstCS1 were marked on the 3D figure using the information of the conserved motif (HGGG and GXSXG motif) from results of the multiple alignment. The 3D structure of EstCS1 through the I-TASSER online server was identified. This system mainly depends on the two methods, the "LOMETS," which identifies a template-based structural simulation by multiple threading approach based on the PDB, and the "BioLiP," which implements a 3D model through protein function database.

\section{Hydrolysis of Tertiary Alcohols}

For the hydrolysis of tertiary alcohol esters, $100 \mu \mathrm{g}$ of EstCS1 was gently blended with the substrate-mixture solutions, phenol red $(2 \mathrm{mg} / \mathrm{ml})$, and each $25 \mathrm{mM}$ of linalyl acetate, $t$-butyl acetate, and $\alpha$-terpinyl acetate in the buffering system based on $20 \mathrm{mM}$ Tris$\mathrm{HCl}$ (pH 8.0) (Ngo et al., 2014). The change in color with $\mathrm{pH}$ shift was recognized after $5,10,30,60$, and $120 \mathrm{~min}$.

\section{Nucleotide Sequence Accession Number}

The nucleotide sequence data of the novel lipolytic gene (estCS1) has been deposited in GenBank (accession number. AEQ63714).

\footnotetext{
${ }^{1}$ https://swissmodel.expasy.org

${ }^{2}$ https://zhanglab.ccmb.med.umich.edu/I-TASSER/

${ }^{3}$ rcsb.org

${ }^{4}$ http://espript.ibcp.fr/ESPript/ESPript/index.php
} 


\section{RESULTS}

\section{Screening and Subcloning of the Putative Esterase Gene}

A metagenomic fosmid library constructed from a compost sample was made to screen clones indicating lipolytic activity with a halo formation around E. coli colonies on LB-tributyrin agar plate. Primarily, 11 lipolytic active clones among $\sim 13,000$ recombinant fosmid clones were obtained, and the one showing the highest positive activity was selected for further studies of characterization. Subcloning of the gene encoding lipolytic activity within the fosmid was performed with pUC19 and DH5 $\alpha$ followed by the digestion of DNA fragments by Sau3AI. After selecting subclones with lipolytic activity, sequence analysis of insert DNA within them was performed and the existence of an open reading frame (designated estCS1) composed of $927 \mathrm{bp}$ that could be translated into a protein of 309 amino acids with a predicted molecular weight of $34 \mathrm{kDa}$ was confirmed. A BLAST search for sequence comparison among related proteins conducted at the NCBI obviously revealed that the amino acid sequence of EstCS1 had the high identities to lipolytic enzymes from various cultured and uncultured bacteria, including esterase and alpha/beta hydrolases from five cultured bacteria (GenBank accession nos. WP 119116380.1, AZV42415.1, WP 067408583.1, WP 067408583.1, and TFG96842.1; identities of $44-59 \%)$, lipases/esterases from two uncultured bacterium (GenBank accession no. AAS77247.1 and ABQ11271.1; identity of $43-46 \%$ ), and a hypothetical protein from one uncultured bacterium (GenBank accession no. AEM45131.1; identity of $45 \%)$. The results of the multiple sequence alignment of EstCS1 with closely related enzymes revealed that there were Ser155-Asp255-His285 residues as the conserved sequence motif of EstCS1. In addition, the catalytic nucleophile serine is at position 155 in the consensus GXSXG pentapeptide and the HGGG motif in the oxyanion hole upstream of the pentapeptide motif; these are characteristics of family IV (HSL family) lipolytic enzymes (Figure 1A; Arpigny and Jaeger, 1999; Kim et al., 2015; Huo et al., 2018; Jayanath et al., 2018; Noby et al., 2018). Phylogenetic analysis using amino acid sequences of EstCS1 and other lipases/esterases also revealed that EstCS1 fell within the cluster including family IV lipolytic enzymes (Figure 1B).

\section{Overexpression and Purification of EstCS1}

The active form of the encoded EstCS1 was overexpressed in the soluble fraction of $E$. coli cells through isopropyl-Dthiogalactopyranoside induction. The yield of $69 \%$ from the soluble fraction was acquired through the purification using a Ni-NTA resin, and then, there has been approximately fourfold increase in the specific activity. After denaturing EstCS1, the result of SDS polyacrylamide gel electrophoresis analysis indicated that the enzyme had a molecular mass of nearly $34 \mathrm{kDa}$. The lipolytic activity of the enzyme was visualized as an apparent band corresponding to the native protein having a size of $34 \mathrm{kDa}$ by a zymogram including an indicator (data not shown).

\section{Biochemical Characterization of EstCS1}

Substrate specificity of EstCS1 toward various lengths of acyl chain was investigated by utilizing pNPEs (C2-C12) as substrates. The esterase EstCS1 demonstrated the highest substrate specificity toward $p$-nitrophenyl propionate (C3) and $p$-nitrophenyl caproate (C6) among pNPEs examined. On the other hand, the hydrolytic activity of EstCS1 toward pNPEs with long chains more than C8 was almost not shown (Figure 2). These results implied that the pNPEs with short-chain acyl groups are good substrates for EstCS1, which is in contrast with the pNPEs having acyl groups longer than C8 (Figure 2).

The effect of temperature on the activity of EstCS1 was examined over a range of $10-80^{\circ} \mathrm{C}$, and the enzyme was clarified to have maximal activity at $50^{\circ} \mathrm{C}$ (Figure 3A). The EstCS1 was lasting its activity up to $60^{\circ} \mathrm{C}$, and its residual activity decreased dramatically in temperatures above $60^{\circ} \mathrm{C}$ (Figure 3A). In terms of the $\mathrm{pH}$, EstCS1 indicated the optimal activity at $\mathrm{pH} 8.0$ and was stable within the $\mathrm{pH}$ range between 5 and 10 (Figure 3B).

\section{Effect of Additives on the Activity of EstCS1}

The activity of EstCS1 dwindled as the level of the concentration of Tweens 20, 40, 60, and 80, Na-deoxycholate, 3-[(3cholamidopropyl) dimethylaminonio]-1-propanesulphonate, Na-taurocholate, and Triton $\times-100$ increased, and it was completely inhibited under the presence of SDS (Supplementary Table S1). $\mathrm{Ca}^{2+}, \mathrm{Mg}^{2+}$, and $\mathrm{Mn}^{2+}$ ions slightly improved the activity of EstCS1, while EstCS1 activity was restrained by the presence of $\mathrm{Cu}^{2+}$ and $\mathrm{Zn}^{2+}$ ions (Supplementary Table S2). To investigate the inhibitory effect on the amino acid residues involved in catalysis, various chemical modifiers were applied to EstCS1. The activity of EstCS1 declined dramatically by phenylmethylsulfonylfluoride, as shown in lipolytic enzymes containing a serine residue in the conserved pentapeptide GXSXG of their active site (Supplementary Table S2; Soror et al., 2007; Jayanath et al., 2018). In the condition with 1$10 \mathrm{mM}$ ethylenediaminetetraacetic acid, the activity of EstCS1 was maintained, which suggested that metal cofactors are not essential for the activity of EstCS1 (Supplementary Table S2). DTT, a strong reducing agent, is generally used to eliminate disulfide bonds within proteins and to hinder cysteine residues of proteins from forming intramolecular and intermolecular disulfide bonds. In the case of EstCS1, the presence of 1 and $5 \mathrm{mM}$ DTT increased its activity, indicating that thiol groups may be crucial for enzyme activity (Côté and Shareck, 2010; Peng et al., 2014). The enzyme activity of EstCS1 was sustained in 1 and $5 \mathrm{mM}$ of 2 -mercaptoethanol, but its activity was inhibited strongly when $10 \mathrm{mM}$ of 2-mercaptoethanol was added (Supplementary Table S2).

\section{The Effect of Organic Solvents on the Activity of EstCS1}

To figure out the effect of solvents on activity of EstCS1, various organic solvents were used at final concentrations of $10,20,30$, and $50 \%(\mathrm{v} / \mathrm{v})$. In the presence of ethanol, 2propanol, acetonitrile, and acetone from $10 \%(\mathrm{v} / \mathrm{v})$ to $30 \%$ 
A

EstCS1

AzV42415.1

TMA49878.1

TMB45453.1

TMA62020.1

TMB17505.1

MSP 42224.1
80

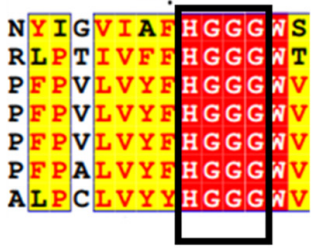

150

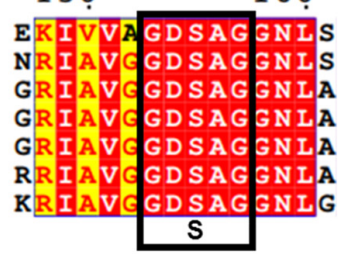

260

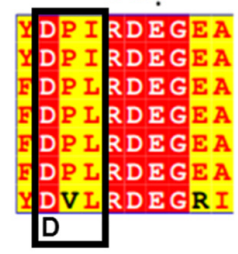

290

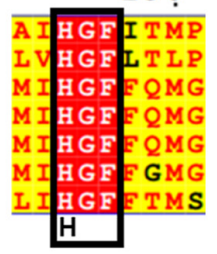

B

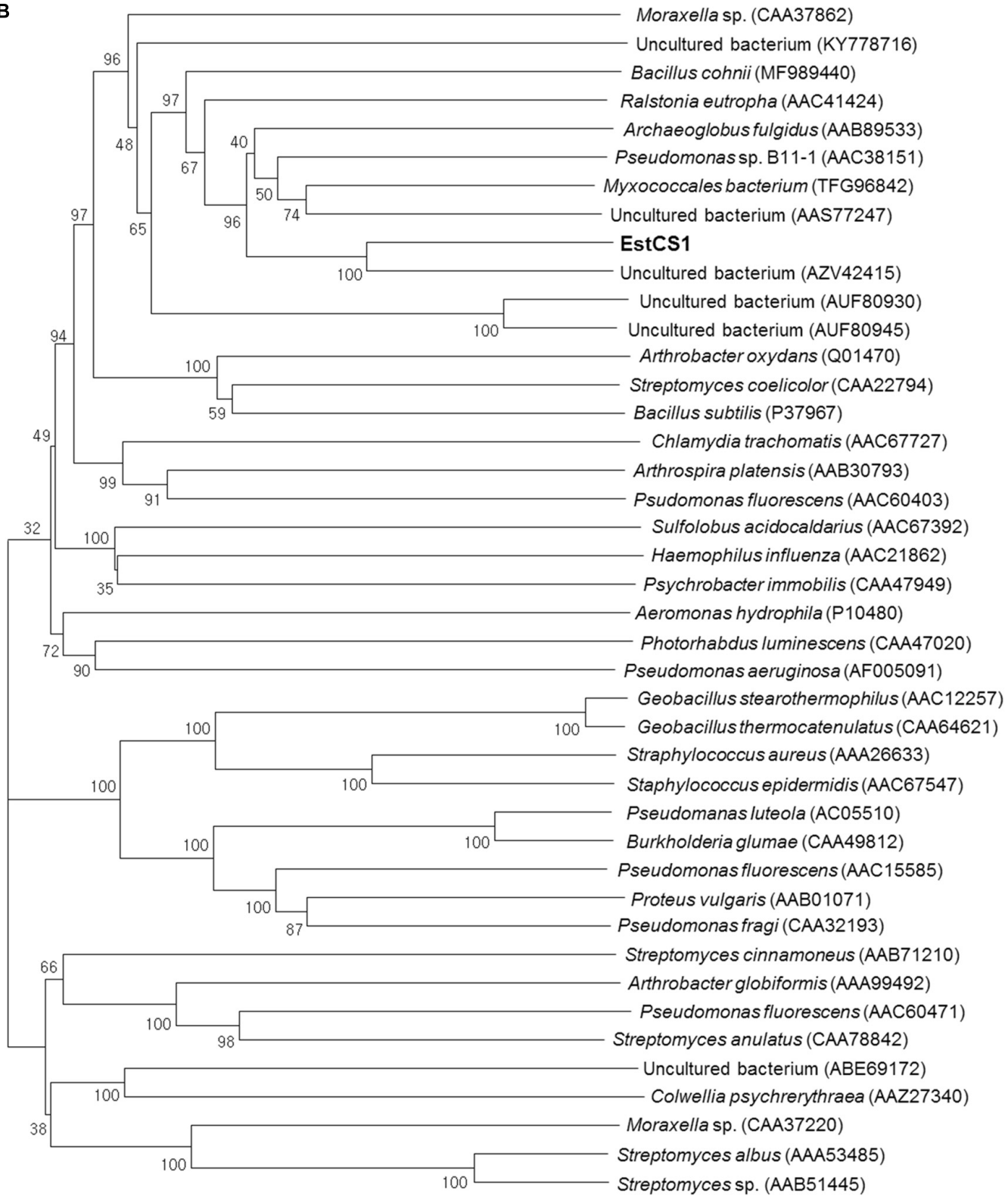

IV

VII

VI

V

II

VIII

II

III

FIGURE 1 | (A) Multiple amino acid sequence alignments of EstCS1 and its homologs. AEM45131.1, hypothetical protein (uncultured organism); AAS77247, esterase lipase (uncultured bacterium); ADR31550, EST1 (uncultured bacterium); ABQ11269, lipase esterase (uncultured bacterium); and ABQ11269, lipase esterase (uncultured bacterium). (B) Phylogenetic tree based on amino acid sequence of EstCS1 and closely related proteins. The phylogenetic tree illustrates a neighbor-joining method using MEGA4. The numbers at the node indicate bootstrap percentages of 1,000 replicates. The units at the bottom of the tree indicate the number of substitution events. Except for EstCS1, the protein sequences for previously identified families of bacterial lipolytic enzymes were retrieved from GenBank (http://www.ncbi.nlm.nih.gov). 


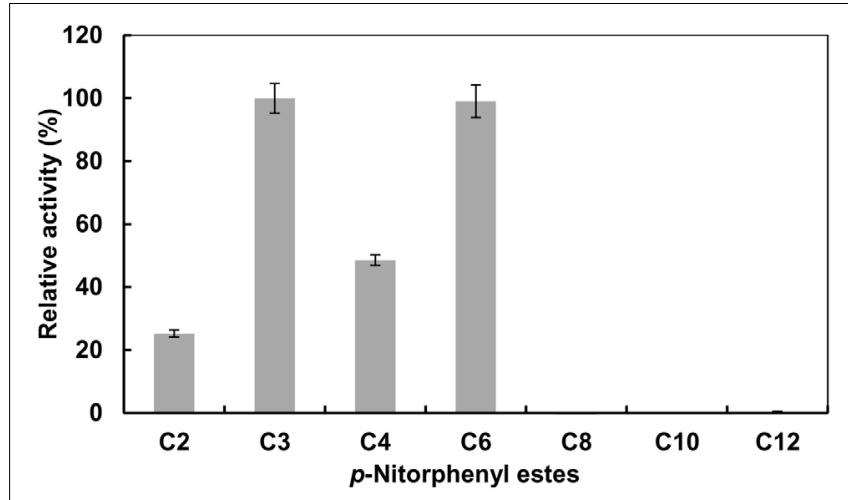

FIGURE 2 | Substrate specificity of EstCS1 toward various $p$-nitrophenyl esters. Substrates used were $p$-nitrophenyl acetate (C2), p-nitrophenyl propionate (C3), p-nitrophenyl butyrate (C4), p-nitrophenyl caproate (C6), $p$-nitrophenyl caprylate (C8), $p$-nitrophenyl caprate (C10), and p-nitrophenyl laurate (C12). The error bars represent mean $\pm \operatorname{SD}(n=3)$.

(v/v), the activity was increased or retained. Whereas enzymatic activity slightly decreased when methanol and DMF from 10\% $(\mathrm{v} / \mathrm{v})$ to $30 \%(\mathrm{v} / \mathrm{v})$ were added (Table 1). The addition of $50 \%(\mathrm{v} / \mathrm{v})$ acetone slightly decreased enzymatic activity, too. However, the addition of $50 \%(\mathrm{v} / \mathrm{v})$ 2-propanol sharply decreased enzymatic activity (Table 1). Other than those, incubation for $1 \mathrm{~h}$ with $50 \%$ (v/v) methanol, acetonitrile, ethanol, DMSO, or DMF showed the enzymatic activity in the range of 43.0$66.1 \%$ (Table 1).

\section{Prediction of 3D Structure, Active Site, and Oxyanion Hole of EstCS1}

Results on the web-based prediction of $3 \mathrm{D}$ structure using the SWISS-MODEL system showed that 50 reference enzymes with structures registered in the PDB (see text footnote 3 ) are related to the sequence information of EstCS1. Among them, the best two predicted models in terms of the identity, coverage, global model quality estimate (GMQE), and qualitative model energy analysis (QMEAN) were derived from 3ZWQ (model 1, sequence identity, 46.88\%; coverage, 0.93; GMQE, 0.73; QMEAN, -2.97) and 4YPV (model 2, sequence identity, 43.75; coverage, 0.93; GMQE, 0.73; QMEAN, -1.91) in the PDB. The model 2 based on the structure of the $4 \mathrm{YPV}$ was assumed to be a monomer, but the predicted structure from 3ZWQ was estimated to be a homo-tetramer. Combining these results with the molecular mass of EstCS1 suggested that model 2 has better predictive structure. In addition, the sequence and position of the conserved motif and the active site of EstCS1 from alignment results of the T-COFFEE and the ESPript 3.0 were identified and then integrated them with model 2 into 3D images using the AntheProt 3D viewer (Figure 4A).

Based on the I-TASSER using different mechanisms for reliable reasoning, model 3 derived from top 4 proteins including 4YPV, 6KMOA, 5JD4, and 1JJI ( $Z$ score: 3.60, 4.36, 0.88, and 1.21, respectively), and its $C$ score was 1.53 . The model 3 was estimated to be a monomer, and it showed the highest TM score in structure with 4YPV through the TM-align structural alignment
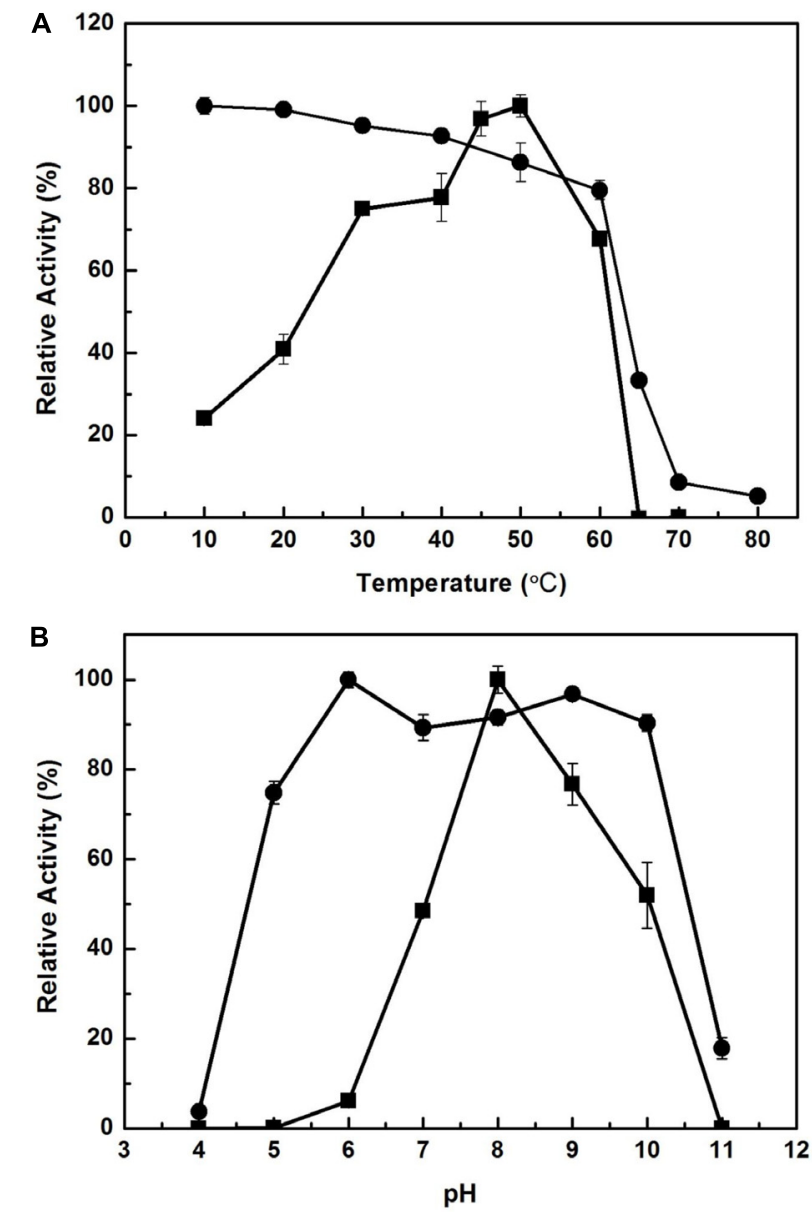

FIGURE 3 | Effect of temperature and pH on the activity of EstCS1. (A) The enzyme activity was measured at each temperature under standard assay conditions $(\mathbf{\square})$. The enzyme was preincubated at the indicated temperatures, and the remaining activity was determined $(\bullet)$. (B) The enzyme activity was measured at each $\mathrm{pH}$ under standard assay conditions $(\boldsymbol{\square})$. The enzyme was preincubated at the indicated $\mathrm{pH}$, and the remaining activity was determined $(\bullet)$.

program (Figure 4B). The prediction of ligand-binding sites showed the highest $C$ score of 0.82 when $3 \mathrm{H} 17 \mathrm{~A}$ data were applied. Besides, when summarizing the top 5 predicted results of ligand-binding sites and active sites, the most importantly anticipated residues were Gly 83 and Gly 84 in the HGGG motif, Ser 155 in the GXSXG motif, Asp 255, and His 285 residues (Figure 4C).

\section{Hydrolysis of Tertiary Alcohol Esters by EstCS1}

The ability of EstCS1 to hydrolyze esters of tertiary alcohols was studied using linalyl acetate, $t$-butyl acetate, and $\alpha$-terpinyl acetate as substrates and by $\mathrm{pH}$ indicator-based colorimetric assay on the release of acetic acid. The results showed that EstCS1 weakly hydrolyzed $t$-butyl acetate and linalyl acetate after incubation for $120 \mathrm{~min}$ (Supplementary Figure S1). 
TABLE 1 | Effects of organic solvents on the EstC1.

\begin{tabular}{lcccc}
\hline Solvent & \multicolumn{4}{c}{$\begin{array}{c}\text { Remaining activity (\%) at various solvent } \\
\text { concentrations (\%) of }\end{array}$} \\
\cline { 2 - 5 } & $\mathbf{1 0}$ & $\mathbf{2 0}$ & $\mathbf{3 0}$ & $\mathbf{5 0}$ \\
\hline Control & 100 & 100 & 100 & 100 \\
Methanol & 95.0 & 92.7 & 90.8 & 43.4 \\
Ethanol & 97.8 & 99.9 & 101.4 & 50.3 \\
2-Propanol & 119.0 & 111.2 & 115.8 & 25.2 \\
Acetonitrile & 95.1 & 110.5 & 101.1 & 43.0 \\
DMSO & 91.8 & 86.0 & 74.4 & 66.1 \\
Acetone & 109.4 & 103.9 & 100.0 & 81.8 \\
DMF & 91.6 & 96.8 & 87.4 & 51.2 \\
\hline
\end{tabular}

\section{DISCUSSION}

The metagenomic library of the compost sample was investigated for screening lipolytic enzymes based on the activity-screening method. A lipolytic enzyme, designated EstCS1, was identified in this study. In view of the phylogenetic tree founded on amino acid sequences, the selected EstCS1 was considered as a member of family IV (Figure 1B). The EstCS1 was found to have novel amino acid sequence showing identities $<59 \%$ to lipolytic enzymes from various cultured and uncultured bacteria. Amino sequence analysis indicated that the EstCS1 contained a putative catalytic triad composed of Ser155-Asp255-His285 and conserved sequence motif of esterase/lipase, GXSXG (Figure 1A; Arpigny and Jaeger, 1999). Two C-terminal conserved motifs, DPLR- and - HGF-, were also clarified in the EstCS1 as shown in some closely related enzymes (Lee et al., 2004; Hong et al., 2007; Nacke et al., 2011; Jiang et al., 2012; Ko et al., 2012; Noby et al., 2018).

EstCS1 had a preference toward shot-chain p-nitrophenyl esters up to $\mathrm{C} 6$ as substrates (Figure 2). EstCS1 indicated optimal activity at $50^{\circ} \mathrm{C}$, and its activity was stably sustained in the temperature range of $10-60^{\circ} \mathrm{C}$ (Figure 3A). According to the thermostability data, EstCs1 was shown to be a moderately thermophilic or thermostable esterase, which has been described in some metagenomic esterases belonging to family IV (Rhee et al., 2005; Choi et al., 2013; Noby et al., 2018). The thermostability of EstCS1 may have originated from a moderately thermophilic or thermostable microorganism mainly existing in compost maintaining high temperatures during the composting process. The activity of EstCS1, meanwhile, was measured at various $\mathrm{pH}$ and indicated optimal activity at $\mathrm{pH}$ 8.0. In addition, over $80 \%$ activity of the enzyme was sustained from $\mathrm{pH} 6.0$ to 10 after incubation for $1 \mathrm{~h}$ (Figure 3B). Given the results so far, EstCS1 is considered as a moderately thermophilic and alkaliphilic esterase (Figure 3).

Biocatalysis are especially advantageous when they improve the substrate solubility in organic solvents and suppress undesired side reactions (Alex et al., 2014; Jayanath et al., 2018). In general, the stability of enzymes in hydrophilic organic solvents is diminished because of the solvent penetration effect deforming the enzyme surface (Yang et al., 2004; Park et al., 2013). The stability of esterases affected by organic solvents is advantageous when they are utilized to biotechnology and industrial fields (Kawata and Ogino, 2010; Jayanath et al., 2018). As proposed in the numerous protein engineering approaches, a directed evolutionary approach was adopted to acquire stable enzymes in hydrophilic organic solvents (Park et al., 2013). In this study, it was clearly revealed that EstCS1 has high stability in polar organic solvents (Table 1). When exposed to methanol, ethanol, acetonitrile, 2-propanol, acetonitrile, DMSO, acetone, and DMF of $30 \%(\mathrm{v} / \mathrm{v})$, the enzyme was shown to retain its activity to over 70\% (Table 1). Besides, EstCS1 turned out to possess an activity of more than $50 \%$ in $50 \%$ ethanol, DMSO, acetone, and DMF (v/v) (Table 1). To our knowledge, there are few studies on enzymes having stability like this in condition with high solvent concentrations of $30-50 \%(\mathrm{v} / \mathrm{v})$ among characterized lipolytic enzymes categorized into the family IV (Jiang et al., 2012; Ko et al., 2012; Oh et al., 2012; Fang et al., 2015; Kim et al., 2015; Huo et al., 2018; Jayanath et al., 2018).

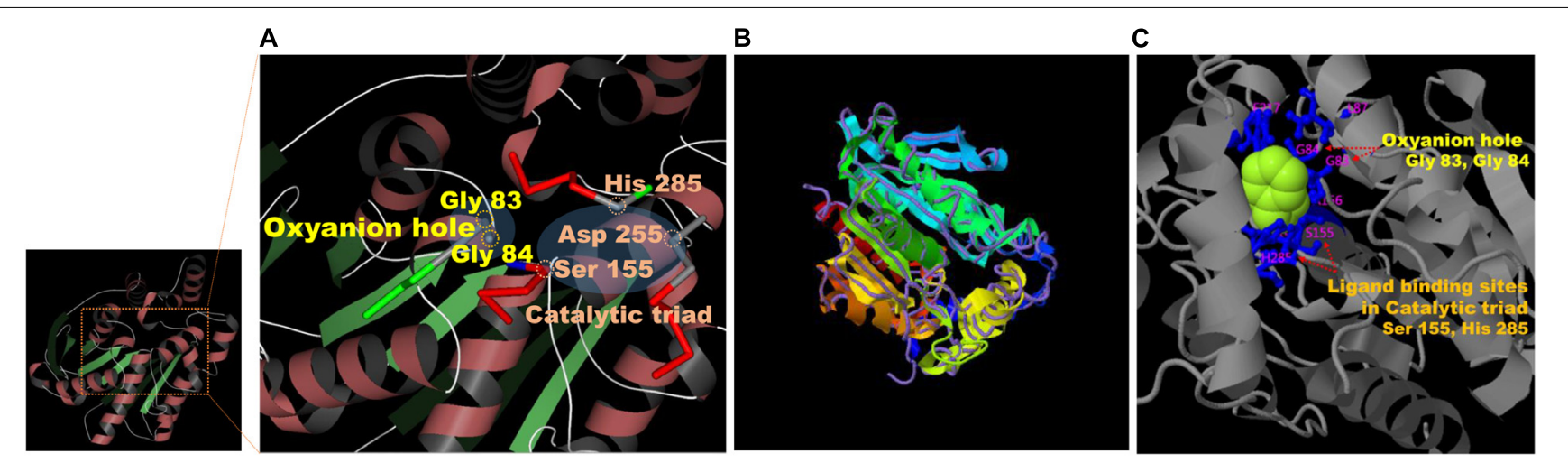

FIGURE 4 | (A) Model 2 structure and major residues based on the analysis of the SWISS-MODEL system (https://swissmodel.expasy.org). (B) The structural prediction of the model 3 (ribbon parameters) with the structurally most analogous protein (4YPV, purple line) in PDB using the I-TASSER system (https://zhanglab.ccmb.med.umich.edu/l-TASSER/). (C) Visualization of the combination between a phenylmethanesulfonic acid (PMS) and the major residues predicted to be important for ligand biding and activity of model 3 from the multiple function annotation analysis of the I-TASSER. 
The ability of EstCS1 to cleave sterically hindered esters between two tertiary alcohols is notable. Given the results of the multiple alignment and structure modeling, EstCS1 shared a GGG(A)X motif in the oxyanion hole close to the active site as shown in most esterases and lipases with the activity at the sterically hindered tertiary alcohols (Henke et al., 2002; Kourist et al., 2007; Jin et al., 2012; Huo et al., 2018; Jayanath et al., 2018). In particular, the residues belonging to the oxyanion hole had been estimated as Gly 83 and Gly 84 in the GGG(A)X motif. They have NH groups forming hydrogen bond with the nucleophilic oxygen of a substrate. The first tetrahedral intermediate of total EstCS1-substrate reaction was created through this chemical combination, which stabilized the negative charge of the transition state. The sequential exchange of an electron among those in the oxyanion hole and the residues including Ser155, Asp255, and His285 in the active site lowered the activation energy necessary for the reaction, which finally push the substrate to the carboxylic acid. Enlarging the active site surrounding the oxyanion hole thereby providing an enlarged substrate binding site was considered as a reason for activity related to GGG(A)X (Henke et al., 2002; Kourist et al., 2007; Huo et al., 2018).

EstCS1 identified from this study was characterized to be a moderately thermophilic esterase that can cleave esters between two tertiary alcohols and has a high stability against organic solvents. Those properties of EstCS1 are worthy of a lot of attention in various biotechnological applications associated with organic solvents, for example, ester synthesis and transesterification reactions. This study also suggests that the metagenomic approach is still worthy of use to identify novel enzymes with high potential for industrial

\section{REFERENCES}

Alex, D., Shainu, A., Pandey, A., and Sukumaran, R. K. (2014). Esterase active in polar organic solvents from the yeast Pseudozyma sp. NII 08165. Enzyme Res. 2014:494682. doi: 10.1155/2014/494682

Arpigny, J. L., and Jaeger, K. E. (1999). Bacterial lipolytic enzymes: classification and properties. Biochem. J. 343, 177-183. doi: 10.1042/bj3430177

Beffa, T., Blanc, M., Lyon, P. F., Vogt, G., Marchiani, M., Fischer, J. L., et al. (1996). Isolation of Thermus strains from hot composts (60 to 80 (C). Appl. Environ. Microbiol. 62, 1723-1727. doi: 10.1128/aem.62.5.1723-1727.1996

Berini, F., Casciello, C., Marcone, G. L., and Marinelli, F. (2017). Metagenomics: novel enzymes from non-culturable microbes. FEMS Microbiol. Lett. 364:fnx211. doi: 10.1093/femsle/fnx211

Bornscheuer, U. T. (2002). Microbial carboxyl esterases: classification, properties and application in biocatalysis. FEMS Microbiol. Rev. 26, 73-81. doi: 10.1111/j. 1574-6976.2002.tb00599.x

Castilla, A., Panizza, P., Rodríguez, D., Bonino, L., Díaz, P., Irazoqui, G., et al. (2017). A novel thermophilic and halophilic esterase from Janibacter sp. R02, the first member of a new lipase family (family XVII). Enzyme Microb. Technol. 98, 86-95. doi: 10.1016/j.enzmictec.2016.12.010

Choi, J. E., Kwon, M. A., Na, H. Y., Hahm, D. H., and Song, J. K. (2013). Isolation and characterization of a metagenome-derived thermoalkaliphilic esterase with high stability over a broad $\mathrm{pH}$ range. Extremophiles $17,1013-1021$. doi: 10.1007/ s00792-013-0583-z

Côté, A., and Shareck, F. (2010). Expression and characterization of a novel heterologous moderately thermostable lipase derived from metagenomics in applications and to expand our perspective of biodiversity for screening novel enzymes.

\section{DATA AVAILABILITY STATEMENT}

The datasets generated for this study can be found in the GenBank/Accession number AEQ63714.

\section{AUTHOR CONTRIBUTIONS}

J-MP performed the experiments of this study and reviewed the manuscript. C-HK performed the experiments of this study. S-MW supported some of the experiments of this study. K-HO discussed the results of this study. J-HY designed this study and wrote the manuscript.

\section{FUNDING}

This work was supported by Basic Science Research Program (Grant no. 2018R1D1A1B07046473) through the National Research Foundation of Korea (NRF) and BK21 plus project funded by the Ministry of Education.

\section{SUPPLEMENTARY MATERIAL}

The Supplementary Material for this article can be found online at: https://www.frontiersin.org/articles/10.3389/fmicb. 2019.03069/full\#supplementary-material

Streptomyces lividans. J. Ind. Microbiol. Biotechnol. 37, 883-891. doi: 10.1007/ s10295-010-0735-734

Fang, Y., Wang, S., Liu, S., and Jiao, Y. (2015). Discovery a novel organic solvent tolerant esterase from Salinispora arenicola CNP193 through genome mining. Int. J. Biol. Macromol. 80, 334-340. doi: 10.1016/j.ijbiomac.2015.06.045

Goyal, S., Dhull, S. K., and Kapoor, K. K. (2005). Chemical and biological changes during composting of different organic wastes and assessment of compost maturity. Bioresour. Technol. 96, 1584-1591. doi: 10.1016/j.biortech.2004.1 2.012

Gupta, R., Gupta, N., and Rathi, P. (2004). Bacterial lipases: an overview of production, purification and biochemical properties. Appl. Microbiol. Biotechnol. 64, 763-781. doi: 10.1007/s00253-004-1568-1568

Henke, E., Pleiss, J., and Bornscheuer, U. T. (2002). Activity of lipases and esterases towards tertiary alcohols: insights into structure-function relationships. Angew Chem Int. Ed. Engl. 41, 3211-3213. doi: 10.1002/1521-3773(20020902)41: 17<3211::aid-anie3211>3.0.co;2-u

Hong, K. S., Lim, H. K., Chung, E. J., Park, E. J., Lee, M. H., Kim, J.-C., et al. (2007). Selection and characterization of forest soil metagenome genes encoding lipolytic enzymes. J. Microbiol. Biotechnol. 17, 1655-1660.

Huo, Y.-Y., Jian, S.-L., Cheng, H., Rong, Z., Cui, H.-L., and Xu, X.-W. (2018). Two novel deep-sea sediment metagenome-derived esterases: residue 199 is the determinant of substrate specificity and preference. Microb. Cell Fact. 17:16. doi: 10.1186/s12934-018-0864-864

Jayanath, G., Mohandas, S. P., Kachiprath, B., Solomon, S., Sajeevan, T. P., Singh, I. S. B., et al. (2018). A novel solvent tolerant esterase of GDSGG motif subfamily from solar saltern through metagenomic approach: recombinant 
expression and characterization. Int. J. Biol. Macromol. 119, 393-401. doi: 10. 1016/j.ijbiomac.2018.06.057

Jiang, X., Xu, X., Huo, Y., Wu, Y., Zhu, X., Zhang, X., et al. (2012). Identification and characterization of novel esterases from a deep-sea sediment metagenome. Arch. Microbiol. 194, 207-214. doi: 10.1007/s00203-011-0745-742

Jin, P., Pei, X., Du, P., Yin, X., Xiong, X., Wu, H., et al. (2012). Overexpression and characterization of a new organic solvent-tolerant esterase derived from soil metagenomic DNA. Bioresour. Technol. 116, 234-240. doi: 10.1016/j.biortech. 2011.10.087

Kawata, T., and Ogino, H. (2010). Amino acid residues involved in organic solventstability of the LST-03 lipase. Biochem. Biophys. Res. Commun. 400, 384-388. doi: 10.1016/j.bbrc.2010.08.080

Kim, E.-Y., Oh, K.-H., Lee, M.-H., Kang, C.-H., Oh, T.-K., and Yoon, J.-H. (2009). Novel cold-adapted alkaline lipase from an intertidal flat metagenome and proposal for a new family of bacterial lipases. Appl. Environ. Microbiol. 75, 257-260. doi: 10.1128/AEM.01400-1408

Kim, H. J., Jeong, Y. S., Jung, W. K., Kim, S. K., Lee, H. W., Kahng, H. Y., et al. (2015). Characterization of novel family IV esterase and family I.3 lipase from an oil-polluted mud flat metagenome. Mol. Biotechnol. 57, 781-792. doi: 10.1007/s12033-015-9871-9874

Kim, T. D. (2017). Bacterial hormone-sensitive lipases (bHSLs): emerging enzymes for biotechnological applications. J. Microbiol. Biotechnol. 27, 1907-1915. doi: 10.4014/jmb.1708.08004

Ko, K.-C., Rim, S.-O., Han, Y., Shin, B. S., Kim, G.-J., Choi, J. H., et al. (2012). Identification and characterization of a novel cold-adapted esterase from a metagenomic library of mountain soil. J. Ind. Microbiol. Biotechnol. 39, 681689. doi: 10.1007/s10295-011-1080-y

Kourist, R., Krishna, S. H., Patel, J. S., Bartnek, F., Hitchman, T. S., Weiner, D. P., et al. (2007). Bornscheuer, Identification of a metagenome-derived esterase with high enantioselectivity in the kinetic resolution of arylaliphatic tertiary alcohols. Org. Biomol. Chem. 5, 3310-3313. doi: 10.1039/B709965G

Kumar, S., Stecher, G., Li, M., Knyaz, C., and Tamura, K. (2018). MEGA X: molecular evolutionary genetics analysis across computing platforms. Mol. Biol. Evol. 35, 1547-1549. doi: 10.1093/molbev/msy096

Lee, M.-H., Lee, C.-H., Oh, T.-K., Song, J.-K., and Yoon, J.-H. (2006). Isolation and characterization of a novel lipase from a metagenomic library of tidal flat sediments: evidence for a new family of bacterial lipases. Appl. Environ. Microbiol. 72, 7406-7409. doi: 10.1128/AEM.01157-1156

Lee, S.-W., Won, K., Kim, H. K., Kim, J.-C., Choi, G. J., and Cho, K. Y. (2004). Screening for novel lipolytic enzymes from uncultured soil microorganisms. Appl. Microbiol. Biotechnol. 65, 720-726. doi: 10.1007/s00253-004-1722-1723

Nacke, H., Will, C., Herzog, S., Nowka, B., Engelhaupt, M., and Daniel, R. (2011). Identification of novel lipolytic genes and gene families by screening of metagenomic libraries derived from soil samples of the German biodiversity exploratories. FEMS Microbiol. Ecol. 78, 188-201. doi: 10.1111/j.1574-6941. 2011.01088.x

Ngo, T. D., Ryu, B. H., Ju, H., Jang, E. J., Kim, K. K., and Kim, T. D. (2014). Crystallographic analysis and biochemical applications of a novel penicillin-bindingprotein/ $\beta$-lactamase homologue from a metagenomic library. Acta Crystallogr. D Biol. Crystallogr. 70, 2455-2466. doi: 10.1107/ S1399004714015272

Noby, N., Saeed, H., Embaby, A. M., Pavlidis, I. V., and Hussein, A. (2018). Cloning, expression and characterization of cold active esterase (EstN7) from Bacillus cohnii strain N1: a novel member of family IV. Int. J. Biol. Macromol. 120, 1247-1255. doi: 10.1016/j.ijbiomac.2018.07.169

Oh, K.-H., Nguyen, G.-S., Kim, E.-Y., Kourist, R., Bornscheuer, U., Oh, T.-K., et al. (2012). Characterization of a novel esterase isolated from intertidal flat metagenome and its tertiary alcohols synthesis. J. Mol. Catal. B Enzym. 80, 67-73. doi: 10.1016/j.molcatb.2012.04.015

Panda, T., and Gowrishankar, B. S. (2005). Production and applications of esterases. Appl. Microbiol. Biotechnol. 67, 160-169. doi: 10.1007/s00253-004-1840-y

Park, H. J., Joo, J. C., Park, K., Kim, Y. H., and Yoo, Y. J. (2013). Prediction of the solvent affecting site and the computational design of stable Candida antarctica lipase B in a hydrophilic organic solvent. J. Biotechol. 163, 346-352. doi: 10.1016/j.jbiotec.2012.11.006

Peng, Q., Wang, X., Shang, M., Huang, J., Guan, G., Li, Y., et al. (2014). Isolation of a novel alkaline-stable lipase from a metagenome library and its specific application for milkfat favor production. Microb. Cell Fact. 13:1. doi: 10.1186/ 1475-2859-13-11

Poli, A., Romano, I., Caliendo, G., Nicolaus, G., Orlando, P., de Falco, A. et al. (2006). Geobacillus toebii subsp. decanicus subsp. nov., a hydrocarbondegrading, heavy metal resistant bacterium from hot compost. J. Gen. Appl. Microbiol. 52, 223-234. doi: 10.2323/jgam.52.223

Privé, F., Newbold, C. J., Kaderbhai, N. N., Girdwood, S. G., Golyshina, O. V., Golyshin, P. N., et al. (2015). Isolation and characterization of novel lipases/esterases from a bovine rumen metagenome. Appl. Microbiol. Biotechnol. 99, 5475-5485. doi: 10.1007/s00253-014-6355-6356

Rhee, J.-K., Ahn, D.-G., Kim, Y.-G., and Oh, J.-W. (2005). New thermophilic and thermostable esterase with sequence similarity to the hormonesensitive lipase family, cloned from a metagenomic library. Appl. Environ. Microbiol. 71, 817-825. doi: 10.1128/AEM.71.2.817-825.2005

Schmeisser, C., Steele, H., and Streit, W. R. (2007). Metagenomics, biotechnology with non-culturable microbes. Appl. Microbiol. Biotechnol. 75, 955-962. doi: 10.1007/s00253-007-0945-945

Soror, S. H., Verma, V., Rao, R., Rasool, S., Koul, S., Qazi, G. N., et al. (2007). A cold-active esterase of Streptomyces coelicolor A3(2): from genome sequence to enzyme activity. J. Ind. Microbiol. Biotechnol. 34, 525-531. doi: 10.1007/s10295007-0224-226

Tutino, M. L., di Prisco, G., Marino, G., and de Pascale, D. (2009). Cold-adapted esterases and lipases: From fundamentals to application. Protein Pept. Lett. 16, 1172-1180. doi: 10.2174/092986609789071270

Uchiyama, T., and Miyazaki, K. (2009). Functional metagenomics for enzyme discovery: challenges to efficient screening. Curr. Opin. Biotechnol. 20, 616-622. doi: 10.1016/j.copbio.2009.09.010

Yang, L., Dordick, J. S., and Garde, S. (2004). Hydration of enzyme in nonaqueous media is consistent with solvent dependence of its activity. Biophys. J. 87, 812-821. doi: 10.1529/biophysj.104.041269

Yeaman, S. J., Smith, G. M., Jepson, C. A., Wood, S. L., and Emmison, N. (1994). The multifunctional role of hormone-sensitive lipase in lipid metabolism. Adv. Enzyme Regul. 34, 355-370. doi: 10.1016/0065-2571(94)90022-90021

Zhou, J., Bruns, M. A., and Tiedje, J. M. (1996). DNA recovery from soils of diverse composition. Appl. Environ. Microbiol. 62, 316-322. doi: 10.1128/aem.62.2. 316-322.1996

Conflict of Interest: The authors declare that the research was conducted in the absence of any commercial or financial relationships that could be construed as a potential conflict of interest.

Copyright (c) 2020 Park, Kang, Won, Oh and Yoon. This is an open-access article distributed under the terms of the Creative Commons Attribution License (CC BY). The use, distribution or reproduction in other forums is permitted, provided the original author(s) and the copyright owner(s) are credited and that the original publication in this journal is cited, in accordance with accepted academic practice. No use, distribution or reproduction is permitted which does not comply with these terms. 